\title{
Learning the min-max gait comfort region when wearing shoes
}

\author{
S. Strada ${ }^{1, *}$, P. Ćorović ${ }^{1}$, C. F. O. da Silva ${ }^{1}$, V. Gabbi ${ }^{1}$, D. Penati ${ }^{1}$, P. Casali ${ }^{2}$, and S. M. Savaresi ${ }^{1}$
}

\begin{abstract}
Shoe-embedded sensors are a recent, common and convenient choice for devices intended for locomotionrelated applications. They are advantageous, compared to other wearable devices, since they allow performing gait assessment in real-world environments, recognizing the user walking pattern in real-time and directly on the feet level. However, shoe with embedded sensors, while providing affore-mentioned advantages, must in no way disturb the walk. In this paper, we present a simple, reliable and cheap method to check whether, and in which manner, the shoe affects the gait. The ultimate goal of the developed methodology is to provide a way to assess the performance of an innovative sole adaptable to changes in the external environment and in the dynamic state of the user. The proposed method is based on using a 2D camera to monitor three geometrical angles (knee, ankle, shoe) which reflect, in a simple but exhaustive manner, the lower limb behavior during the gait cycle. The ability of the shoe not to affect the normal gait is tested by comparison between the three angles trends of a person wearing the shoe and the person's min-max gait comfort regions, with respect to the three angles. Min-max comfort regions are learned through real experiments on different subjects and shoes.
\end{abstract}

\section{INTRODUCTION AND PROBLEM STATEMENT}

Walking is the natural and instinctive form for the human being to move and is essential for daily life activities and social participation. Gait analysis is widely accepted as a general measure of functional ability among individuals as it is related to health, well-being and quality of life. To be able to detect deviations or changes from healthy and normal walking, it is necessary to have a reference gait behavior available of healthy and young subjects. The human gait can be analyzed from several points of view and specialties, in terms of gait cycle spatiotemporal structure, specific lower limb joints' kinematics, more detailed electromyography acquisitions, just to mention the most exploited features [1].

Usual clinical investigations to highlight the possible presence of pathologies rely on acquiring and analyzing the many different gait related quantities when a individual walks barefoot in dedicated and sensorized ambients. The fact of requiring the patient to walk barefoot is of fundamental importance as the objective, in the medical context, is to highlight possible pathologies, which clearly can manifest themselves as clearly as possible through walking with naked feet. On the other hand, the aim of this study is rather to

\footnotetext{
${ }^{1}$ Silvia Strada, Davide Penati, Vittorio Gabbi, Caio Fabio Oliveira da Silva, Pavle Ćorović and Sergio Savaresi are with the Dipartimento di Elettronica, Informazione e Bioingegneria, Politecnico di Milano, Piazza Leonardo da Vinci 32, 20133 Milan, Italy. Email: silvia.strada, sergio.savaresiepolimi.it

${ }^{2}$ Patrizia Casali is with E-Novia, Via San Martino 12, Milan 20122, Italy. Email: patrizia.casali@e-novia.it

* corresponding author
}

characterize a healthy and comfortable gait pattern whilst wearing athletic shoes or runners, so defining the "normality" walk region in young adults and establishing a methodology for future testing of sound and safe footware. Moreover, the methodology presented in this paper has the ultimate goal of providing a very simple and fast tool, based on easy instrumentation and sensors and on measured data easy to interpret even in the industrial sector, for testing the new sensored and actuated Wahu shoe. It consists of a shoe with a sole that is adaptable to changes in the external environment and in the dynamic state of the user but, of course, it is of paramount importance that this footware does not impact negatively one's gait but, on the contrary, has a beneficial action overall.

Thus, the ultimate purpose of our work is to measure, through simple walking trials, lower limb angles - ankle, knee and an angle related to footware itself, whose precise definition will be explained later - and to define admissible min-max ranges for the aforementioned parameters of human gait with shoes. To the best of the authors' knowledge, the latter goal was not explored in the literature before. The influence of footware on walking is treated in [2], whereas a system based on measurements using insole sensors is illustrated in [3]. Then, there exist some approaches based either on Kinect cameras [4], two or more high frame rate cameras [5], or on a single high frame rate camera [6]. We based our study on the latter device, whose accuracy has been assessed in [7] and whose effectiveness has been proven and will be described throughout the paper. It is worth mentioning that some manufacturers, such as Vicon, have commercial motion capture systems for gait analysis, which however often require completely dedicated spaces and hardware/software tools.

\section{FEATURES FOR GAIT CHARACTERIZATION}

This Section is dedicated to a brief, but sufficiently exhaustive, description of the practicable ways an individual's gait can be characterized. Subsequently, the features considered most appropriate for outlining the walking pattern while wearing shoes will be described in detail, together with the sensing equipment to measure them in real time.

Gait analysis is of fundamental importance and usually has the objective of providing clinicians with detailed quantitative information capable of characterizing the gait of pathological subjects, as well as their variations with respect to a normal situation. However, the evaluation of the characteristics of posture and movement can be of enormous utility also for simply monitoring how a person is walking, 
in daily life or doing sport, in these cases not barefoot but wearing footwear (Fig 1).

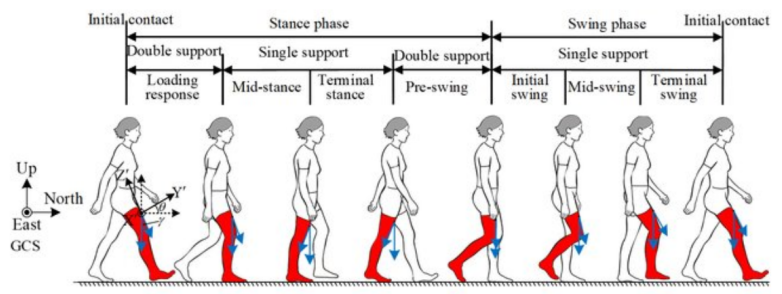

Fig. 1: Human gait characteristics. Typical gait events and gait phases.

Quantitative description of human gait aims to quantitatively describe a particular walking pattern. Typically, human gait can be analyzed by highlighting: its temporal and spatial parameters and/or its kinematic and kinetic parameters [1]:

- temporal and spatial gait parameters are the easiest to measure, e.g. to estimate step length, step width, walking speed, stride time and other, only a stopwatch and a meter are necessary.

- more advanced methods and techniques are preferred because of higher precision, robustness and repeatability of measurements. Kinematic parameters include mainly angles and rotations of anatomic joints and body segments that are analysed. Kinetic parameters give information about the dynamics of the movement and include forces and pressures applied to the sole/floor or within the muscles during the movement.

To the first class of parameters, as already outlied, belong features such as step length, stride, or also different types of angles involved in the walking process. In our study we have decided to focus on the former category of parameters, discarding both kinematic and kinetic metrics, mainly due to the following two reasons. Regarding muscles' activity, these measurements are mostly relevant in clinically related applications [1], whereas highlighting forces and pressures requires a much more costly experimental setup which is certainly not needed for the characterization of a healthy person's walk.

The spatial parameters considered in this paper are listed in Table I. The ankle and knee angle, described in detail later in this Section, are among the most often tracked and the most relevant gait parameters, [8], both in healthy and in patological subjects applications. The shoe angle is a new time varying angle needed to represent the influence of the footwear on the gait. The aforementioned three key angles are graphicaly highlighted on the image of a leg in Fig. 2.

Here it is worth noting that other typical spatial parameters, such as speed, pace or stride length, were not measured for the need to contain the costs of the sensing instrumentation. However, our method could be extended, with slight modifications, to incorporate also their measurement and their exploitation.

The knee angle is the angle related to the movement of the knee. This articulation is an extremelly complex joint,

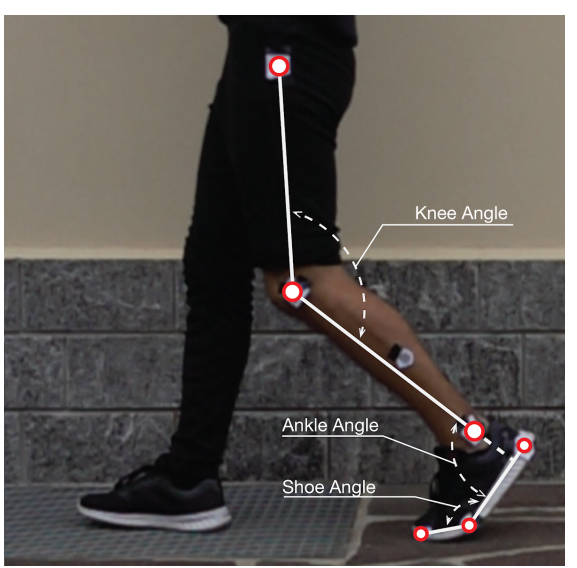

Fig. 2: Measured angles

\begin{tabular}{ll} 
Angle & Description \\
\hline \hline Knee angle & Angle between tibia and femur \\
\hline Ankle angle & Angle between tibia and foot \\
\hline Shoe angle & Angle determining the shoe bending \\
\hline
\end{tabular}

TABLE I: Spatial parameters

that has three degrees of freedom. However, most of the movement is done in sagittal plane and that movement will be measured here. There are two basic motions occuring: flexion (back of the tibia is moving towards the back of the femur) and extension (back of the tibia is moving from the back of the femur).

The related angle is measured between tibia and femur. Tibia is measured as the line connecting the knee and tibiotalar joint, while femur is measured as the line connecting the knee with the hip. After the measurement, the angle is normalized so that the straight leg corresponds to the zero angle.

Healthy shape of the knee angle in barefoot walking during the whole gait cycle is shown in Fig. 3. Deviation from the healthy region might be a symptom of a pathology, such as quadriceps weakness, quadriceps spasticity, hip flexor weakness, knee valgus/varus [1].

The ankle angle is the angle connected to the movement of the ankle. Ankle's motion is secured by three joints subtalar, midtarsal and tibiotalar (Fig. 4). Even though the overall ankle's motion is guaranteed by the movement of all three joints, the tibiotalar one certainly plays the most important role, [1]. Two basic movements occur: dorsiflexion (ankle angle decreases as the foot is moving closer to the leg) and plantar flexion (ankle angle increases as the foot is moving further from the leg). It is worth noting that these are most widely used anatomical terms, but equivalent synonyms can be found in the literature.

The ankle angle is physically the angle between tibia and foot. As before, the tibia is schematically represented as a line connecting the knee and the tibiotalar joint, while the foot is simplified as an arbitrary line along the foot itself. After being measured, the ankle angle can be normalized 


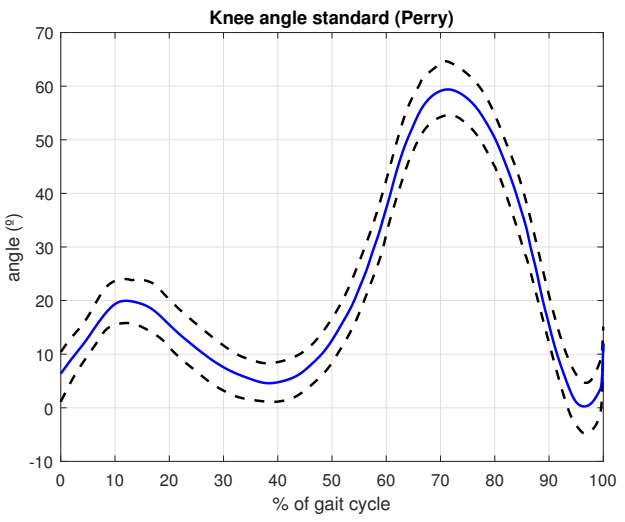

Fig. 3: Knee angle during the gait cycle, on healthy, barefoot subjects; the full line represents the average, while the dotted lines are the envelope of the normal, healthy region [1]

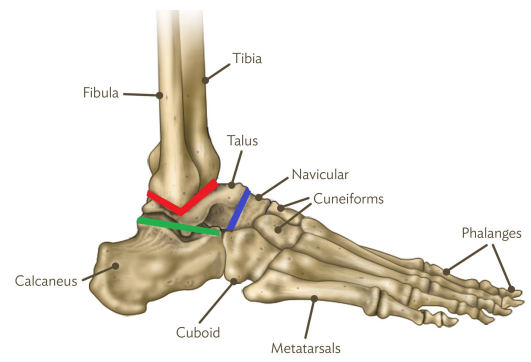

Fig. 4: Joints in the ankle region: red - tibiotalar joint, blue - midtarsal joint, green - subtalar joint

in some way. Normalization is important because the line representing the foot can be chosen arbitrarily, so different measurements can lead to the trends that keep the shape of the angle, but possibly have an offset. However, after the normalization, the results are consistent even when the line representing the foot is chosen in a different manner, [9]. This is especially important in case of measuring angles with footwear present, since it is hard to identify the same line in the foot when the foot is covered with different shoes. In this paper, the line representing the foot was chosen in the same way in all the experiments, so the measerements were consistent without normalization.

An example of healthy shape of the ankle angle in a healthy, barefoot walking during the whole gait cycle is shown in Fig. 5. Consequently, various forms of deviation from the healthy region might be a symptom of a pathology, such as soleus spasticity, gastrocnemius spasticity, tibial muscle weakness and soleus weakness or others, [1].

Finally, the shoe angle, as shown in Fig. 2, is the "new" feature that is introduced to incorporate in our setup the monitoring of the footware influence on gait behavior. This angle is meant to represent footwear rigidity. Since it is not measured on the person, but on the shoe, we cannot make direct conclusions about the health of the subject based on this angle. However, this angle reflects the shoe characteristics and its performance during walking, thus affecting all other angles and gait itself, and obviously ultimately one's

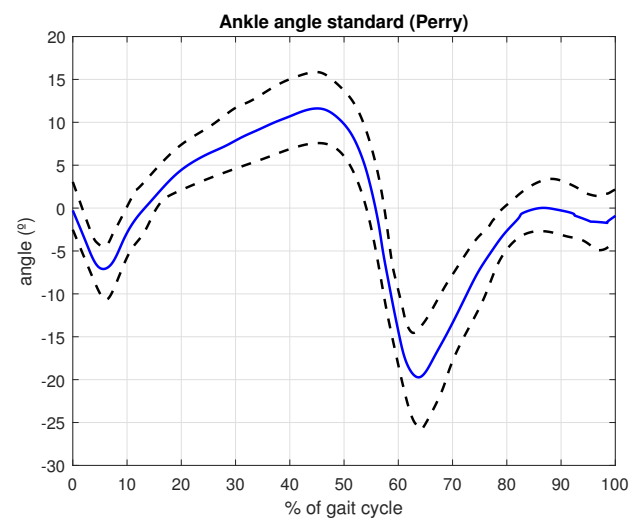

Fig. 5: Time behavior of the ankle angle during a gait cycle, in healthy, barefoot subjects; the full line represents the average evolution, while the dotted lines are the envelope of the the healthy region, [1]

walk comfort.

The shoe angle measures the angle between the foot (taken as the line connecting the heel and the location where the shoe is bent) and the toes (represented by the line connecting the tip of the shoe and the location where the shoe is bent).

We will show later on in this paper examples of healthy shapes of the shoe angle during the whole gait cycle. In fact, the main contribution of this paper comprises the definition of a healthy and footware-based standard gait through the time analysis of the defined three key angles. One of them is new and here we will assess its behavior, while the other two angles are known in the literature and in the practice but mainly on barefoot subjects.

\section{EXPERIMENTAL SETUP FOR 2D GAIT ANALYSIS}

Recall that objective of our work is the development of a sensing equipment and its data analysis framework for standard gait caharcterization when wearing shoes. Therefore the measurement systems and calculation methods must be as agile, cost-effective and computationally light as possible. In our research work, we evaluated different settings and at the end converged on an low cost experimental setup, which is described in this Section and depicted in Fig. 6.

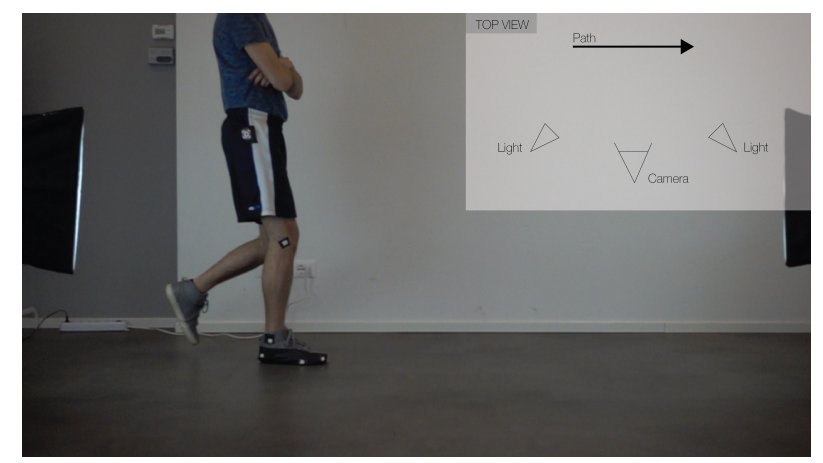

Fig. 6: The experimental setup - a 2D camera view and the room top view 
For medical investigation purposes, gait analysis is usually performed in a controlled environment, possibly equipped with several cameras (3D and 2D) and/or different kinds of additional sensors, often force/pressure sensing devices embedded in the floor. After many feasibility tests, we came up with the need of just one 2D camera for the monitoring of the three above mentioned angles during walking. Then, our methodology falls into the class of 2D gait analysis procedures, though having the innovative issue of analyzing the human walk when wearing footware.

In the experiments different healthy subjects are asked to follow a straight path perpendicular to the camera view axis. Only one side of the subject is visible in each frame and only one leg is considered, while the other one is partially covered during the motion. Passive markers are positioned on the subject's leg in order to measure the minimal set of features which we established are the most affected by the shoes the subject was wearing.

In detail, the experimental setup consists of:

- 6 Markers: according to Section II, markers are positioned on the hip, knee and ankle, whereas three other markers are positioned on the shoe: the first on the metatarsal head, the second on the shoe heel and the last marker was placed approximatively in the middle point of the shoe, in correspondence of its bending point in the longitudinal direction.

- a 2D Camera: it must be set up in order to record at a high frame rate in order to capture each phase of the gait with sufficient time resolution. Specifically, 500 frames per second and a shutter speed of $1 \mathrm{~ms}$. allow for no motion blur along the full sequence of frames. In order to achieve the maximum resolution possible for each marker only one gait cycle is recorded for each video. Specifically, the video starts and ends when the heel touches the ground. The subjects are asked to walk at a normal speed taking around 1,5 s. to cover the full cycle.

- a graphics software: the recorded video is imported into an open source graphics software named Blender. This software provides a specific tool for motion tracking analysis that is used for detecting automatically each marker, within each frame. At the end, Blender creates a two columns .csv file containing the markers' $x$ and $\mathrm{y}$ coordinates (in pixels), each frame being associated to a row.

- a data analysis environment: The .csv files from Blender are imported into Matlab and there elaborated according to the algorithmic procedure described in the next Section.

\section{AlgORITHM FOR STANDARD GAIT REGION SHAPING}

In this section, we will explain the procedure for converting the markers' positions, obtained as explained in section III, to the angle trends. Using the 6 markers' positions (hip, knee, tibiotalar joint, heel, midfoot, toetip), we can define 4 body segments as the vectors connecting them: tibia, femur, foot, toes, as shown in section II. Then, we aim to calculate the angles between the body segments' vectors.

The positions of markers are brought into the system as points in three-dimensional space, where first coordinate corresponds to their position along the width of the frame, second coordinate coresponds to their position along the height of the frame, and third coordinate is always equal to zero. Vectors representing the body segments are then taken as the three-dimensional vectors connecting these points.

For understanding the procedure, take knee angle. The relevant body segments to this calculation are femur and tibia. Define the vector representing femur $\vec{V}_{\text {femur }}$ as a vector starting from the knee and ending in the hip, and the vector representing tibia $\vec{V}_{t i b i a}$ as the vector starting in the knee and ending in the tibiotalar joint.

Now, define corresponding unitary direction vectors:

$$
\vec{V}_{\text {tibia } 0}=\frac{\vec{V}_{\text {tibia }}}{\left|\vec{V}_{\text {tibia }}\right|} \quad \vec{V}_{\text {femur } 0}=\frac{\vec{V}_{\text {femur }}}{\left|\vec{V}_{\text {femur }}\right|}
$$

Then, the angle between them can be calculated as:

$$
\phi_{\text {knee }}=\operatorname{atan} 2\left(\left|\vec{V}_{\text {femur } 0} \times \vec{V}_{\text {tibia } 0}\right|, \vec{V}_{\text {femur } 0} \circ \vec{V}_{\text {tibia } 0}\right)
$$

The similar procedure can be used for the other two angles. To generalize, there are three steps:

1) define vectors of the body segment, oriented so that they start in the center of the measured angle

2) calculate vectors of unitary intensity with the direction of the body segment vectors

3) calculate the desired angle as atan 2 of the intensity of the cross-product of the unitary vectors as the first operand, and their dot product as the second one

After the angles are calculated, they can, if needed, be normalized in different ways. In this paper, foot angle is not normalized, while knee and ankle angle are normalized, as explained in section II, using formulas:

$$
\phi_{\text {knee, }, \text { norm }}=-\phi_{\text {knee }} \quad \phi_{\text {ankle, }, \text { norm }}=\frac{\pi}{2}-\phi_{\text {ankle }}
$$

Using this procedure, all three relevant trends can be obtained.

Problems with analyzing the obtained data can be due to the different lenghts of trends (given that the subjects walk at their natural pace, which is not the same for all). For this reason, all the trends are "aligned" by making them into the same length trends, using methods of stretching and interpolating.

\section{EXPERIMENTAL RESULTS}

The real experiments, executed to collect the data, were conducted on three healthy subjects each wearing four different shoes. For each shoe, five to seven trials of the experiment were performed on the same subject, so information on the pair subject-shoe could be gathered redundantly ensuring sufficient reliability. Moreover, the pairs of shoes used, see Tab. II, were quite different from each other, thus making it possible to represent, to a good extent, the effects of some 


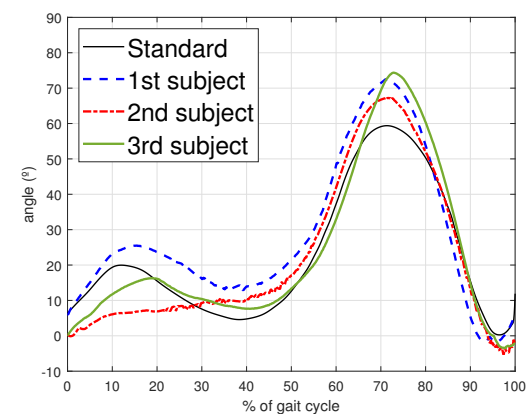

(a) Knee angle.

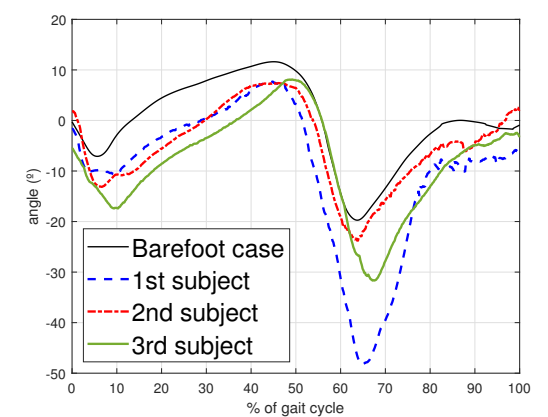

(b) Ankle angle.

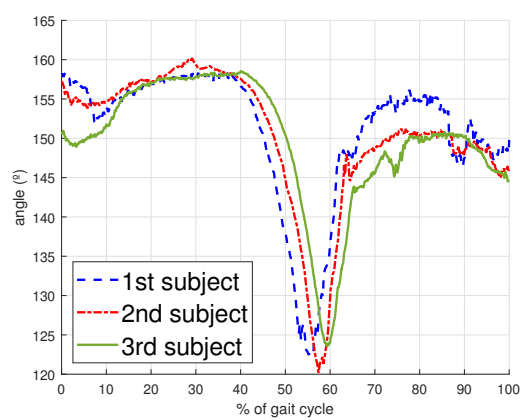

(c) Shoe angle.

Fig. 7: Average knee, ankle, and shoe angles for each subject, considering all shoes. The knee and ankle angles are compared to their respective "standard" barefoot cases found in the literature.

\begin{tabular}{l|llll} 
Shoes characteristics & \multicolumn{3}{l}{} \\
\hline \hline Image & $\begin{array}{l}\text { Weight } \\
(\mathbf{g})\end{array}$ & $\begin{array}{l}\text { Sole height } \\
(\mathbf{c m})\end{array}$ & $\begin{array}{l}\text { Height } \\
(\mathrm{cm})\end{array}$ & $\begin{array}{l}\text { Width } \\
(\mathbf{c m})\end{array}$ \\
\hline & 350 & 2.8 & 9 & 7 \\
& 232 & 3 & 10 & 8 \\
& 442 & 2 & 13.5 & 7 \\
\hline
\end{tabular}

TABLE II: Characteristics (average values) of the used shoes: weight, sole height, maximum height of the shoe from the ground, with no one wearing it, and minimum width.

popular sneakers on the walking pattern. Markers positions were captured by the camera for each experiment and then transformed into angle trends, following the steps shown in Section IV. Concerning the knee and ankle angles, averaged over over the four different shoe models, and reported in Figs. $7 \mathrm{a}$ and $7 \mathrm{~b}$, the shape of these graphs is coherent with that known from the literature for the barefoot case, but vary considerably in amplitude due to the fact that the person wears shoes. Moreover, from Fig. 7, we observe that each subject exploits the same pattern over the gait but each curve differs from the other two. The differences in amplitude and time shift are, at this extent, admissible and can be attributed to the unique gait motion of each individual, and biomechanical composition of the person, e.g., height, weight, and length of the bones. For the shoe angle behavior, Fig. 7c, interestingly enough, we can depict a completely new information on its individual and inter-subject variability and shape. The shoe angle, though its variability among different individuals, exhibits a characteristic valley soon after half of the gait cycle, with two almost flat sections before and after. A small time shift can be observed among the three curves for all the three angles. More specifically, the time shift can be clearly noticed by comparing the time instants where the knee angle curves assume their respective maximum values. Correspondingly, the same applies to the minimum values of the ankle and shoe functions. A probable cause of this

\begin{tabular}{l|lll}
\multicolumn{2}{l}{ Range of motion (degrees) } & & \\
\hline \hline & Knee & Ankle & Shoe \\
\hline 1st subject & 65,48 & 68,01 & 49,94 \\
2nd subject & 67,33 & 40,24 & 52,14 \\
3rd subject & 71,08 & 49,31 & 45,91 \\
\hline
\end{tabular}

TABLE III: Range of motion for each subject-angle pair.

offset is the fact that the subjects walked at their "natural speed" without limitations, so that, in processing the data and resorting to interporlation, i.e. to considering as abscissa the percentage of the gait cycle (from $0 \%$ to $100 \%$ ) instead of time, each event happens at a slightly different instant during one's walk. The range of variation, here defined as the difference between maximum and minimum angles within the whole cycle, is shown in Table III for each subject. In particular, the ankle angle of the first subject varies the most relative to those of the others. This is illustrated by Fig. $7 \mathrm{~b}$, where in the pre-swing phase, the first subject exhibits significantly more plantar flexion, that is, the curve assumes the lowest values in this region. In Fig. 8 the behavior of the knee, ankle and shoe angles are depicted for the three different subjects, where each subject walking characteristics are averaged on the different shoes.

In order to define a sort of "normal" walk region, for every test subject and for each of the three indicator angles, an area in each angle's plane is defined and highlighted only for the first subject, for the sake of compactness, in light blue in Fig. 8. To this areas belong all gait functions of the specific measured angle, in repeated normal gaits wearing sequentially all four models of shoes. The aformentioned shaded areas are delimited by an lower and upper limiting curves, which can be seen as the min-max gait comfort bounds, consisting of the minimum and maximum angles for each percentage "instant" of the gait cycle over the different footware. Hence, each shaded area, gives an easy to define, individual admissible sector for his/her normal gait angles when shoes are on the feet. These admissible sectors are learned from one's gait trough experiments with different shoes, but, once this reference bounds are calculated for 


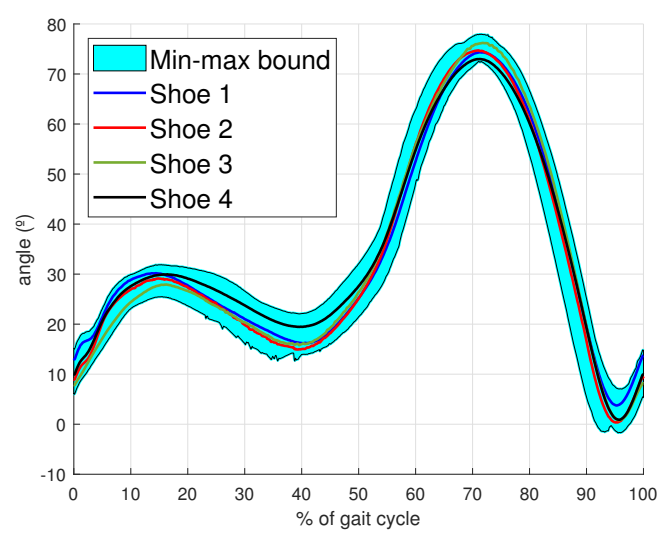

(a) Knee angle.

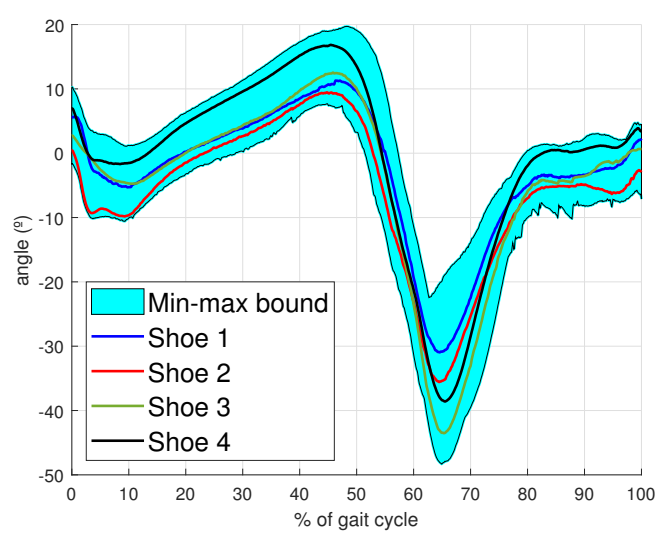

(b) Ankle angle.

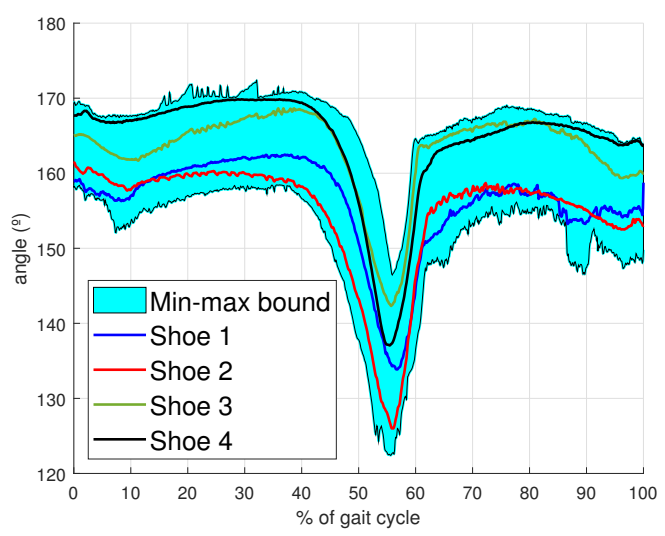

(c) Shoe angle.

Fig. 8: Average knee, ankle and shoe angles of one of the tested subjects wearing four differents shoes. The min-max bounds and areas are displayed as the shaded blue areas.

a person, they should represent his/her "shoe-independent" footprint of his/her healthy way of walking and can serve as a standard for the testing of new shoes.

\section{CONCLUSION AND FUTURE WORK}

In this paper, a low-cost and reliable methodology is outlined for the definition of a "standard" gait cycle of a healthy person wearing footware. The standard gait is linked to the definition of three spatial-temporal parameters, which are geometrically defined on the leg and denominated the knee, ankle, and shoe angles. The latter being a comfort indicator, introduced in this paper and not found in literature, whose role is to represent the shoe sole's flexibility. The interdependence of the three angles has still to be investigated, though not being within the scope of the present research since not the medical nalysis and diagnosis of gait is the issue but rather the definition of a healthy set of gaits. The three angles are measured with a simple 2D camera detecting passive leg markers positions at high frequency and, compared to most of the many different gait parameters, are specifically useful for comfort and health assessments of shoes, which is the main motivation for this research. The experimental setup is relatively simple, particularly when compared to the typical gait analysis infrastructure reported in the pertinent literature, which in almost all cases pertains diagnosis and treatment of patological cases. It is worth stressing that the proposed methodology aims mainly at examining the impact of shoes on the subject's gait at early stages, and possibly indicate the need for more detailed experiments. Hence, no stronger claims about the comfort or health risks can be stated solely based on the methodology described herein. Accordingly, the method stands as an extra tool that can be used to perform simple, but informative preliminary experiments. In a future research, a more comprehensive and holistic analysis is recommended, for instance, quantitative and qualitative methods, such as, pressure profile analysis, tridimensional visual analysis, and the subjective reports of individuals, after long-term use of the shoes. Moreover, further work has to focus on the definition of a statistically meaningful min-max region which will surely require a much wider test campaign on different subjects, in order to establish the relevant distribution and calculate statistical parameters.

\section{REFERENCES}

[1] J. Perry, Gait Analysis, Normal and Pathological Gait. SLACK Incorporated, 1992.

[2] C. Morio, M. J. Lake, N. Gueguen, G. Rao, and L. Baly, "The influence of footwear on foot motion during walking and running," Journal of Biomechanics, vol. 42, no. 13, pp. 2081 - 2088, 2009.

[3] J. A. Ramirez-Bautista, J. A. Huerta-Ruelas, S. L. Chaparro-Cárdenas, and A. Hernández-Zavala, "A review in detection and monitoring gait disorders using in-shoe plantar measurement systems," IEEE Reviews in Biomedical Engineering, vol. 10, pp. 299-309, 2017.

[4] E. Cippitelli, S. Gasparrini, S. Spinsante, and E. Gambi, "Kinect as a tool for gait analysis: Validation of a real-time joint extraction algorithm working in side view," Sensors, vol. 15, no. 1, pp. 1417-1434, 2015.

[5] Yu-Ren Li, Shaou-Gang Miaou, C. K. Hung, and Julius Tube Sese, "A gait analysis system using two cameras with orthogonal view," in 2011 International Conference on Multimedia Technology, pp. 2841-2844, 2011.

[6] P. Soda, A. Carta, D. Formica, and E. Guglielmelli, "A low-cost videobased tool for clinical gait analysis," in 2009 Annual International Conference of the IEEE Engineering in Medicine and Biology Society, pp. 3979-3982, 2009.

[7] U. C. Ugbolue, E. Papi, K. T. Kaliarntas, A. Kerr, L. Earl, V. M. Pomeroy, and P. J. Rowe, "The evaluation of an inexpensive, $2 \mathrm{~d}$, video based gait assessment system for clinical use," Gait \& Posture, no. 38, pp. 483-489, 2013.

[8] M. Roberts, D. Mongeon, and F. Prince, "Biomechanical parameters for gait analysis: a system review of healthy human gait," Physical Therapy and Rehabilitation, vol. 4, no. 6, pp. 1-17, 2017.

[9] M. W. Whittle, Gait Analysis, an Introduction. Elsevier, Ltd, 2007. 